\title{
Installment Options and Static Hedging
}

\author{
MARK H. A. DAVIS \\ Department of Mathematics, Imperial College, \\ London SW7 2BZ, England \\ Walter Schachermayer and Robert G. Tompkins \\ Financial and Actuarial Mathematics Group, \\ Technische Universität Wien \\ Wiedner Hauptstrasse 8, A-1040 Wien, Austria
}

January 8, 2001

\begin{abstract}
An installment option is a European option in which the premium, instead of being paid up-front, is paid in a series of installments. If all installments are paid the holder receives the exercise value, but the holder has the right to terminate payments on any payment date, in which case the option lapses with no further payments on either side. We discuss pricing and risk management for these options, in particular the use of static hedges to obtain both no-arbitrage pricing bounds and very effective hedging strategies with almost no vega risk.
\end{abstract}

\section{Introduction}

In a conventional option contract the buyer pays the premium up front and acquires the right, but not the obligation, to exercise the option at a fixed time $T$ in the future (for European-style exercise) or at any time at or before $T$ (for American-style exercise). In this paper we consider an alternative form of contract in which the buyer pays a smaller up-front premium and then a sequence of "installments", i.e. further premium payments at equally spaced time intervals before the maturity time $T$. If all installments are paid the buyer can exercise the option, European-style, at time $T$. Crucially, though, the buyer has the right to 'walk away': if any installment is not paid then the contract terminates with no further payments on either side. This provides useful extra optionality to the buyer while, as we shall see, the seller can hedge the option using simple static hedges that largely eliminate model risk.

The case of two installments is equivalent to a compound option, previously considered by Geske [7] and Selby and Hodges [8]. Let $C(t, T, S, K)$ denote the Black-Scholes value at time $t$ of a European call option with strike $K$ maturing at time $T$ when the current underlying price is $S$ (all other model parameters are constant). Installments $p_{0}, p_{1}$ are paid at times $t_{0}, t_{1}$ and final exercise is at time $T>t_{1}$. At time $t_{1}$ the holder can either pay the premium $p_{1}$ and continue to hold the option, or walk away, so the value at $t_{1}$ is $\max \left(C\left(t_{1}, T, S\left(t_{1}\right), K\right)-p_{1}, 0\right)$. The holder will pay the premium $p_{1}$ if this is less than the value of the call 
option. The value of this contract at $t_{0}$ is thus the value of a call on $C$ with 'strike' $p_{1}$.

Another way of looking at it, that will be useful later, is this: the holder buys the underlying call at time $t_{0}$ for a premium $p=p_{0}+e^{-r\left(t_{1}-t_{0}\right)} p_{1}$ (the NPV of the two premium payments where $r$ denotes the riskless interest rate) but has the right to sell the option at time $t_{1}$ for price $p_{1}$. The compound call is thus equivalent to the underlying call option plus a put on the call with exercise at time $t_{1}$ and strike price $p_{1}$. The value $p$ is thus greater than the Black-Scholes value $C\left(t_{0}, T, S\left(t_{0}\right), K\right)$, the difference being the value of the put on the call.

A similar analysis applies to installment options with premium payments $p_{0}, p_{1}, \ldots, p_{k}$ at times $t_{0}, \ldots, t_{k}<T$. The NPV of the premium payments is $p=\sum_{i=0}^{k} p_{i} e^{-r\left(t_{i}-t_{0}\right)}$ and the installment option is equivalent to paying $p$ at time $t_{0}$ and acquiring the underlying option plus the right to sell it at time $t_{j}, 1 \leq j \leq k$ at a price $q_{j}=\sum_{i=j}^{k} p_{i} e^{-r\left(t_{i}-t_{j}\right)}$ (all subsequent premiums are 'refunded' when the right to sell is exercised). The installment option is thus equivalent to the underlying option plus a Bermuda put on the underlying option with time-varying strike $q_{i}$.

In the next section we discuss pricing in the Black-Scholes framework. As for American options a finite-difference algorithm must be used. In section 3 , simply-stated 'no-arbitrage' bounds on the price are derived valid for very general price process models. As will be seen, these depend on comparison with other options and suggest possible classes of hedging strategies. In section 4 we introduce and analyse static hedges for installment options, concentrating on a specific example to illustrate our point. Concluding remarks are given in Section 5.

This paper is largely a summary of our companion paper [5], which also contains a discussion of 'continuous-installment options', not considered here.

\section{Pricing in the Black-Scholes framework}

Consider an asset whose price process $S_{t}$ is the conventional log-normal diffusion

$$
d S_{t}=r S_{t} d t+\sigma S_{t} d w_{t},
$$

where $r$ is the riskless rate and $w_{t}$ a standard Brownian motion; thus (1) is the price process in the risk-neutral measure. We consider a European call option on $S_{t}$ with exercise time $T$ and payoff

$$
\left[S_{T}-K\right]^{+}=\max \left(S_{T}-K, 0\right) .
$$

The Black-Scholes value of this option at time 0 is of course

$$
p_{\mathrm{BS}}=E e^{-r T}\left[S_{T}-K\right]^{+} .
$$

$p_{\mathrm{BS}}$ is the unique arbitrage-free price for the option, to be paid at time 0 . As an illustrative example we will take $T=1$ year, $r=0, K=100, S_{0}=100$ and $\sigma=25.132 \%$, giving $p_{\mathrm{BS}}=10.00$.

In an installment option we choose times $0=t_{0}<t_{1}<\cdots<t_{n}=T$ (generally $t_{i}=i T / n$ to a close approximation). We pay an upfront premium $p_{0}$ at $t_{0}$ and pay an 'installment' of $p_{1}$ at each of the $n-1$ times $t_{1}, \ldots, t_{n-1}$. We also have the right to walk away from the deal at each time $t_{i}$ : if the installment 
due at $t_{i}$ is not paid then the deal is terminated with no further payments on either side. The pricing problem is to determine what is the no arbitrage value of the premium $p_{1}$ for a given value of $p_{0}$. The present value of premium payments - assuming they are all paid - is

$$
p_{0}+p_{1} \sum_{i=1}^{n-1} e^{-r t_{i}}
$$

and this must exceed the Black-Scholes value in view of the extra optionality.

Computing the exact value is straightforward in principle. Let $V_{i}(S)$ denote the net value of the deal to the holder at time $t_{i}$ when the asset price is $S_{t_{i}}=S$. In particular

$$
V_{n}(S)=[S-K]^{+} .
$$

At time $t_{i}$ we can either walk away, or pay $p_{1}$ to continue, the continuation value being

$$
E_{t_{i}, S\left(t_{i}\right)}\left[e^{-r\left(t_{i+1}-t_{i}\right)} V_{i+1}\left(S_{t_{i+1}}\right)\right] .
$$

Thus

$$
V_{i}(S)=\max \left(0, E_{t_{i}, S}\left[e^{-r\left(t_{i+1}-t_{i}\right)} V_{i+1}\left(S_{t_{i+1}}\right)\right]-p_{1}\right) .
$$

In particular, $V_{n-1}$ is just the maximum of 0 and $\mathrm{BS}-p_{1}$, where BS denotes the Black-Scholes value of the option at time $t_{n-1}$. The unique arbitrage free value of the initial premium is then

$$
p_{0}=V_{0}^{+}\left(S_{0}, p_{1}\right):=E_{t_{0}, S_{0}}\left[e^{-r\left(t_{1}-t_{0}\right)} V_{1}\left(S_{t_{1}}\right)\right] .
$$

For fixed $p_{1}, V_{0}^{+}\left(S_{0}, p_{1}\right)$ is easily evaluated using a binomial or trinomial tree and this determines the up-front payment $p_{0}$. If we want to go the other way round, pre-specifying $p_{0}$, then we need a simple one-dimensional search to solve the equation $p_{0}=V_{0}^{+}\left(S_{0}, p_{1}\right)$ for $p_{1}$. A similar search solves the equation $\widehat{p}=V_{0}^{+}\left(S_{0}, \widehat{p}\right)$ giving the installment value $\widehat{p}$ when all installments, including the initial one, are the same.

Figure 1 shows the price $\widehat{p}$ at time 0 for our standard example with 4 equal installments. For comparison, one quarter of the Black-Scholes value is also shown. At $S_{0}=100, \widehat{p}=3.284$, which is $31 \%$ greater than one quarter of the Black-Scholes value. Figure 2 shows the value at time $t_{1}$; when $S\left(t_{1}\right) \leq 98.28$ it is optimal not to continue and the option has value zero.

\section{$3 \quad$ No-arbitrage bounds derived from static hedges}

The pricing model of the previous section makes the standard Black-Scholes assumptions: log-normal price process, constant volatility. By considering static super-replicating portfolios, however, we can determine easily computable bounds on the price valid for essentially arbitrary price models. We need only assume that for any $s \in\left[t_{0}, T\right]$ there is a liquid market for European calls with maturities $t \in[s, T]$, the price being given by

$$
C(s, t, K)=\mathbb{E}_{Q}\left[e^{-r(t-s)}\left(S_{t}-K\right)^{+} \mid \mathcal{F}_{s}\right]
$$




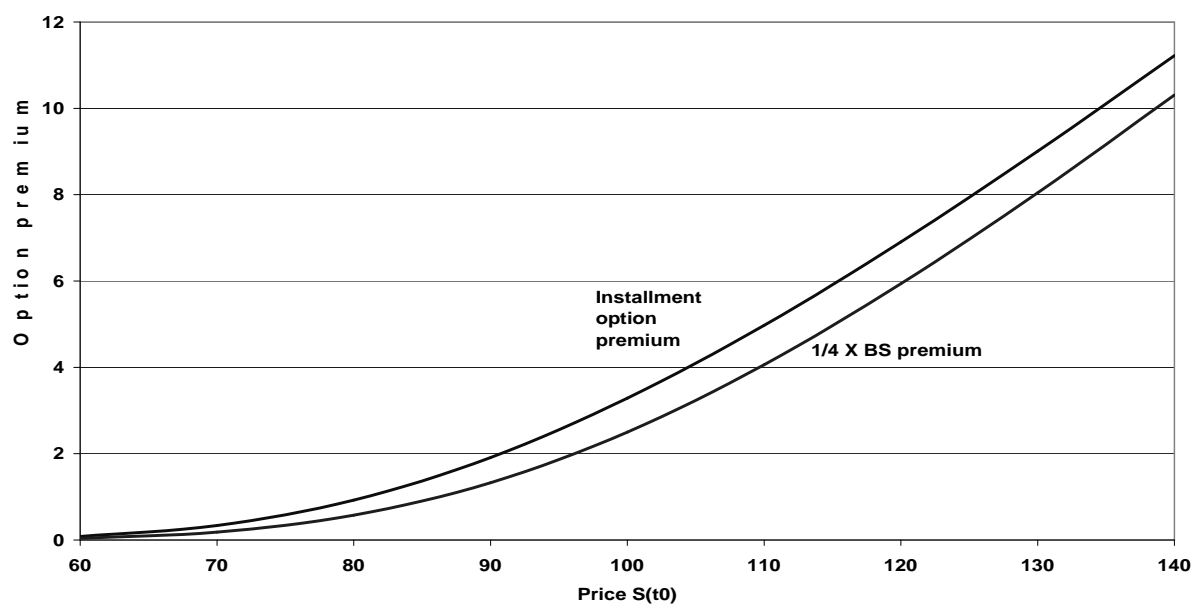

Figure 1: Fair installment value and Black-Scholes value

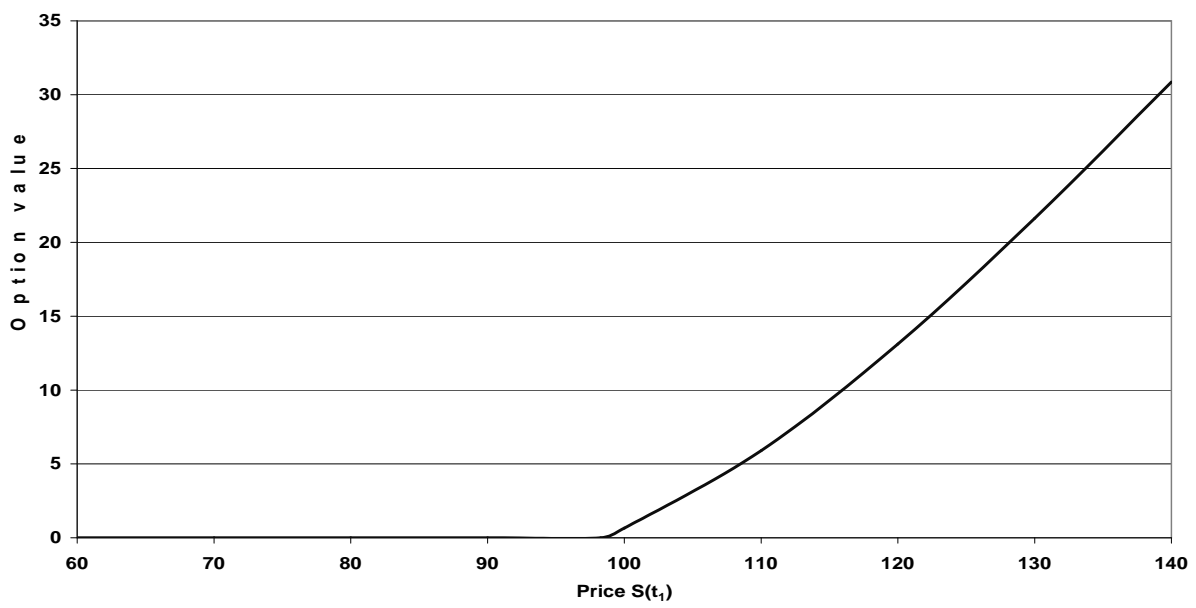

Figure 2: Value of installment option at time $t_{1}$ as function of price $S\left(t_{1}\right)$

where $Q$ is a martingale measure for the process $S$ and $\mathcal{F}_{s}$ denotes the information available at time $s$. By put-call parity this also determines the value of put options $P(s, t, K)$. We know today's prices $C\left(t_{0}, t, K\right)$ and that is all we know about the process $S$ and the measure $Q$. We ignore interest rate volatility, assuming for notational convenience that the riskless rate is a constant, $r$, in continuously compounding terms. We also assume that no dividends are paid.

Let us first consider a 2-installment, i.e. compound, option, with premiums $p_{0}, p_{1}$ paid at $t_{0}, t_{1}$ for an underlying option with strike $K$ maturing at $T=t_{2}$.

The subsequent result provides no-arbitrage bounds on the prices $p_{0}, p_{1}$ which are independent of the special choice of the model $S$ and the equivalent martingale measure $Q$.

Proposition 3.1 For the compound option described above, there is an arbitrage opportunity if $p_{0}, p_{1}$ do not satisfy the inequalities

$$
C\left(t_{0}, T, K+e^{r\left(T-t_{1}\right)} p_{1}\right)>p_{0}>C\left(t_{0}, T, K\right)-e^{-r\left(t_{1}-t_{0}\right)} p_{1}+P\left(t_{0}, t_{1}, p_{1}\right) .
$$


Proof. Denote $K^{\prime}=K+e^{r\left(T-t_{1}\right)} p_{1}$ and suppose we sell the compound option with agreed premium payments $p_{0}, p_{1}$ such that $p_{0} \geq C\left(t_{0}, T, K^{\prime}\right)$. We then buy the call with strike $K^{\prime}$ and place $x=p_{0}-C\left(t_{0}, T, K^{\prime}\right) \geq 0$ in the riskless account. If the second installment is not paid, the value of our position at time $t_{1}$ is $x e^{r\left(t_{1}-t_{0}\right)}+C\left(t_{1}, T, K^{\prime}\right) \geq 0$, whereas if the second installment is paid we add it to the cash account, and the value at time $T$ is then $x e^{r\left(T-t_{0}\right)}+$ $p_{1} e^{r\left(T-t_{1}\right)}+C\left(T, T, K^{\prime}\right)-C(T, T, K) \geq 0$. This is an arbitrage opportunity, giving the left-hand inequality in (10).

Now suppose the compound option is available at $p_{0}, p_{1}$ satisfying

$$
p_{0}+e^{-r\left(t_{1}-t_{0}\right)} p_{1} \leq C\left(t_{0}, T, K\right)+P\left(t_{0}, t_{1}, p_{1}\right) .
$$

We buy it, i.e. pay $p_{0}$, and sell the two options on the right (call them $\widehat{C}, \widehat{P}$ ), so our cash position is $\widehat{C}+\widehat{P}-p_{0} \geq e^{-r\left(t_{1}-t_{0}\right)} p_{1}$. At time $t_{1}$ the cash position is therefore $x \geq p_{1}$, and we have the right to pay $p_{1}$ and receive the call option. We exercise this right if $C\left(t_{1}, T, K\right) \geq p_{1}$. Then our cash position is $x-p_{1} \geq 0$, $\widehat{C}$ is covered and $\widehat{P}$ will not be exercised because $p_{1}<C\left(t_{1}, T, K\right) \leq S\left(t_{1}\right)$ (the call option value is never greater than the value of the underlying asset). On the other hand, $\max \left(p_{1}-C\left(t_{1}, T, K\right), 0\right) \geq \max \left(p_{1}-S\left(t_{1}\right), 0\right)$, so if $p_{1}>C\left(t_{1}, T, K\right)$ we do not pay the second installment and still have enough cash to cover $\widehat{C}$ and $\widehat{P}$. Thus there is an arbitrage opportunity when the right-hand inequality in (10) is violated.

Of course, for practical purpose $P\left(t_{0}, t_{1}, p_{1}\right)$ will be a negligible quantity (and there will be no liquid market as typically $p_{1} \ll S_{0}$ ) but for obtaining theoretically sharp bounds one must not forget this term.

In fact, the above inequalities are sharp: it is not hard to construct examples of arbitrage-free markets such that the differences in the left (resp. right) inequality in (10) become arbitrarily small.

Finally let us interpret the right hand side of inequality (10) by using the interpretation of the compound option given in the introductory section: the net present value $p_{0}+e^{-r\left(t_{1}-t_{0}\right)} p_{1}$ of the payment for the compound must equal by no-arbitrage - the price $C\left(t_{0}, T, K\right)$ of the corresponding European option plus a put option to sell this call option at time $t_{1}$ at price $p_{1}$. Denoting the latter security by Put(Call) we obtain the no-arbitrage equality.

$$
p_{0}=C\left(t_{0}, T, K\right)-e^{-r\left(t_{1}-t_{0}\right)} p_{1}+\operatorname{Put}(\text { Call }) .
$$

In the proof of proposition 3.1 we have (trivially) estimated this Put on the Call from below by the corresponding Put $P\left(t_{0}, t_{1}, p_{1}\right)$ on the underlying $S$. We now see that the difference in the right hand inequality of $(10)$ is precisely equal to the difference $\operatorname{Put}(\mathrm{Call})-P\left(t_{0}, t_{1}, p_{1}\right)$ in this estimation.

Similar arguments apply for $n$ installments, where holding the installment option is equivalent to holding the underlying option plus the right to sell this option at any installment date at a price equal to the NPV of all future installments. The value of the Bermuda option on the option is greater than the equivalent option on the stock. This gives us the following result.

Proposition 3.2 For the $n$-installment call option with premium payment $p_{0}$ at time $t_{0}$ and $p_{1}$ at times $t_{1}, \ldots, t_{n-1}$ there is an arbitrage opportunity if $p_{0}$, 
$p_{1}$ do not satisfy

$$
C\left(t_{0}, T, K+\widehat{p}_{1}\right)>p_{0}>\left[C\left(t_{0}, T, K\right)-e^{r\left(T-t_{0}\right)} \widehat{p}_{1}+P_{\mathrm{Berm}}\left(t_{0}\right)\right]_{+} .
$$

Here

$$
\widehat{p}_{1}=p_{1} \sum_{i=1}^{n-1} e^{r\left(T-t_{i}\right)}
$$

and $P_{\text {Berm }}\left(t_{0}\right)$ denotes the price at time $t_{0}$ of a Bermuda put option on the underlying $S(t)$ with exercise times $t_{1}, \ldots, t_{n-1}$ and strike price $K_{i}$ at time $t_{i}$, where

$$
K_{i}=p_{1} \sum_{j=i}^{n-1} e^{-r\left(t_{j}-t_{i}\right)}
$$

\section{$4 \quad$ Static Hedging}

Static hedging is a technique that has come increasingly into favour in recent years because of its robustness to market friction and model error. See $[1],[2],[3],[6]$ and [9], for example.

In Section 2 the pricing formula for installment options was derived under the perfect market assumptions made by Black and Scholes. The price is intimately associated with the construction of a riskless dynamic hedging portfolio. In Section 3 we saw that - without making any assumptions about the price process model - arbitrage opportunities exist if the installment option price fails to satisfy certain bounds, derived by comparison with the values of static portfolios of plain vanilla options. In this section our objective is to show that if the price does lie within these bounds then a static hedging strategy based on these portfolios nevertheless provides an excellent hedge. Typically, we find that with these hedges the installment option writer faces a maximum loss that is bounded, with probability 1 , by a number that is a modest fraction - say $20 \%$ - of the original option premium.

In this paper we shall just analyse one example, in order to convey the ideas and convince the reader that static hedges are potentially very effective. The companion paper [5] gives much more evidence, based on extensive simulation under alternative assumptions about the price process, including stochastic volatility and market friction in the form of transaction costs.

Our test case will be the standard example of Section 2 with two equal installments, paid at times $t_{0}=0, t_{1}=0.5$. (This is simply a compound option). Recall that the Black-Scholes value of the underlying plain vanilla option is $p_{\mathrm{BS}}=10.00$. The Black-Scholes value of equal installment premiums is $p_{0}=$ $p_{1}=5.855$. We noted in Section 3 that there would be an arbitrage opportunity if the first installment $p_{0}$ were enough to buy a 1-year call option with strike $K^{\prime}=K+p_{1}=105.855$. In fact the value of this option is $p^{\prime}=7.627$, so there is - of course - no arbitrage. To construct our hedge, we receive $p_{0}$ as premium, borrow the difference $p^{\prime}-p_{0}=1.772$ and buy the $K^{\prime}$-call. The second installment will not be paid if $S\left(t_{1}\right) \leq 97.59$, and in this case we close out the position at $t_{1}$; otherwise the hedge is held to maturity. The curve labelled 'Hedge 1 ' in Figure 3 shows the P\&L of the hedged position at $t_{1}$ as a function of the price $S\left(t_{1}\right)$. The P\&L is equal to $\min \left\{p_{0}-p^{\prime}+C^{\prime}, p_{0}-p^{\prime}+C^{\prime}+p_{1}-C\right\}$, where 
$C^{\prime}=C\left(t_{1}, T, S\left(t_{1}\right), K^{\prime}\right), C=C\left(t_{1}, T, S\left(t_{1}\right), K\right)$. The maximum loss is $p^{\prime}-p_{0}$, which is $17.72 \%$ of the Black-Scholes premium for the underlying option, and the maximum gain is $20.3 \%$ of this premium, realized when the price is on the continuation boundary. Figure 4 shows the distribution of P\&L under the riskneutral measure; this turns out to be close to the uniform distribution. Of course this distribution would be different under different price modelling assumptions, but the main point is that under any reasonable model the expected $\mathrm{P} \& \mathrm{~L}$ is close to zero and the maximum loss is confined to the amount borrowed, which is not very much.

We can do better. Looking at the P\&L profile for Hedge 1 in Figure 3 we note that it is very similar to the value of a calendar spread (the difference between two call options with the same strike but different maturities). This suggests that instead of borrowing 1.772 from the bank to finance purchase of the $K^{\prime}$ call we should raise the money by selling a calendar spread. After some experimentation we find that a suitable calendar spread has strike 97.59 (equal to the price at which the P\&L of Hedge 1 is maximum) and maturity times $T_{1}=0.5, T_{2}=1.0$. The Black-Scholes value of this calendar spread at time 0 is 2.865 , so we need to sell $0.619=1.772 / 2.865$ of them to finance purchase of the $K^{\prime}$ call. We thus create a hedge whose value at time 0 is $p_{0}$ consisting of the following plain vanilla call options $((K, T)$ denotes strike and maturity): long $(97.59,0.5)$ and $(105.86,1.0)$, short $(97.59,1.0)$. The P\&L profile of this hedge at $t_{1}$ is 'Hedge 2' in Figure 3. It is a considerable improvement: the maximum loss has been reduced to $5.1 \%$ of the underlying Black-Scholes premium.

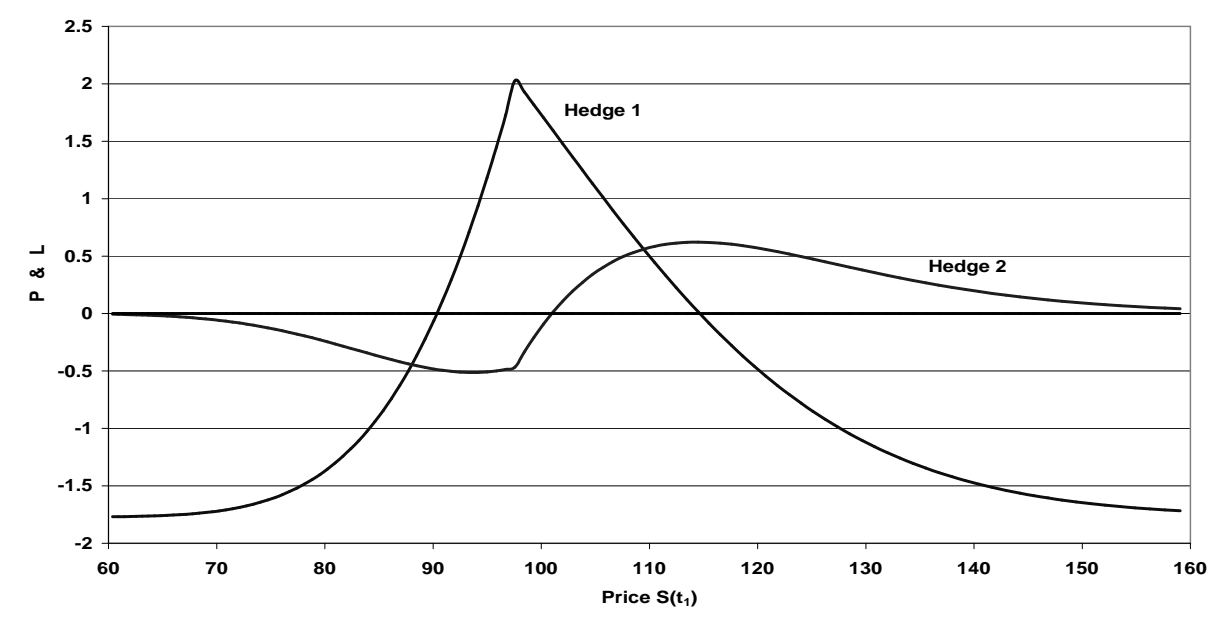

Figure 3: Profit/Loss profiles of compound option static hedges as functions of the underlying asset price $S\left(t_{1}\right)$

\section{Concluding Remarks}

The reader will find in the companion paper [5] a much more complete analysis of static hedges for installment options - including ones with multiple installments - examining examining the performance relative to dynamic hedging under realistic market scenarios and including the effects of transaction costs 


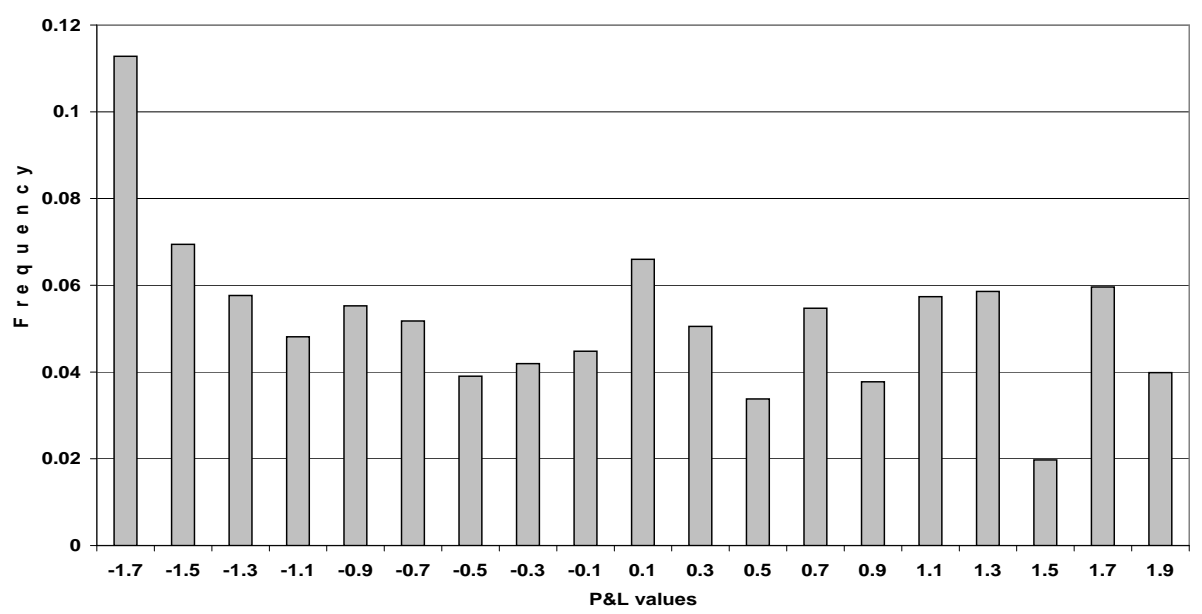

Figure 4: Distribution of Static Hedge PधLL under the risk neutral measure

and stochastic volatility. This study confirms what is suggested by the example presented above, namely that static hedging is a highly effective technique that decouples the hedger almost entirely from the malign effects of market friction and vega risk.

The construction of Hedge 2 in Section 4 - which was frankly done by trial and error - suggests that we should take a more systematic approach. Effectively, plain vanilla option payoffs are being used as basis functions, in terms of which we seek to approximate the payoff profile of an exotic option. While expansion of option payoffs in Fourier series has been investigated by Desrolles and Laurent [4], we are not aware of any studies in which other option payoffs are used as basis functions, although such an approach would not be hard to implement and was in fact suggested to the authors years ago by Michael Selby. We plan to return to this topic.

\section{References}

[1] P. Carr, K. Ellis and V. Gupta, (1998), Static Hedging of Exotic Options. Journal of Finance, Vol. 53, No. 3, pp. 1165-1190.

[2] P. Carr and J.-F. Picron, (1999), Static Hedging of Timing Risk. The Journal of Derivatives, Vol. 6, No. 3, pp. 57-70.

[3] A. Chou and G. Georgiev, (1998), A Uniform Approach to Static Replication. The Journal of Risk, Vol. 1, No. 1, pp. 73-87.

[4] S. Darolles and J.-P. Laurent, (2000), Approximating payoffs and pricing formulas. Journal of Economic Dynamics and Control, Vol. 24, pp. 17211746.

[5] M. Davis, W. Schachermayer and R. Tompkins,(2000), Pricing, no-arbitrage bounds and robust hedging of installment options. Preprint, Technische Universität Wien. 
[6] E. Derman, D. Ergener and I. Kani, (1995), Static Options Replication. The Journal of Derivatives, Vol. 2, No. 4, pp. 78-95.

[7] R. Geske, (1979), The valuation of compound options. Journal of Financial Economics, Vol. 7, pp. 63-81.

[8] M.J.P. Selby, S.D. Hodges, (1987), On the evaluation of compound options. Management Science, Vol. 33, pp. 347-355.

[9] R. Tompkins, (1997), Static versus dynamic hedging of exotic options: an evaluation of hedge performance via simulation. Netexposure, Vol. 1, No. 2, pp. 1-28. 